\title{
LE GROUPE ÉPISCOPAL DE BORDEAUX (GIRONDE)
}

\author{
Wandel MIGEON
}

\begin{abstract}
Mots-clés. Groupe épiscopal, cathédrale paléochrétienne, castrum, Antiquité tardive, Constantin.
Résumé. Les travaux de construction du tramway de Bordeaux ont mis au jour une abside à pans coupés à proximité immédiate de la cathédrale Saint-André, dans l'angle sud-est de l'enceinte antique, érigée aux alentours de 280 apr. J.-C. Les plans, les dimensions de l'édifice et la richesse de son décor nous incitent à voir dans ce monument une construction majeure de Burdigala érigée à la fin du IV s. Il s'agit vraisemblablement de la première cathédrale de Bordeaux érigée au sein d'un groupe épiscopal enfoui sous l'actuelle cathédrale. Avec la basilique Saint-Seurin et la basilique Sainte-Marie toute proche, cet imposant monument révèle l'ancienneté des origines chrétiennes de Bordeaux.
\end{abstract}

Key-words. Bishopric, Early Christian cathedral, castrum, late Antiquity, Constantine.

Abstract. During the road works for the tram at Bordeaux, an apse with cut-off corners was discovered near the cathedral Saint-Andre, at the south-east angle of the Roman wall, built around 280 AD. The plan and size of the building, and the rich ornamentation allow to see in this building a major construction of Burdigala built at the end of the $4^{\text {th }} c$. It must be the first cathedral established inside a bishopric buried underneath the cathedral. With Saint-Seurin basilica and the nearby Sainte-Marie basilica, this impressive monument makes clear the early Christian origins of Bordeaux.

Translation: Isabelle FAUDUET

Schlagwörter. Kirchenfamilie, frühchristliche Kathedrale, castrum, Spätantike, Konstantin.

Zusammenfassung. Bei Vorarbeiten für den Bau einer Straßenbahnlinie wurde in Bordeaux in unmittelbarer Nähe der Kathedrale Saint-André eine polygonale Apsis aufgedeckt. Sie liegt innerhalb der Südostecke der antiken Stadtbefestigung, die um $280 \mathrm{n}$. Chr. errichtet wurde. Entwurf und Ausmaße der Anlage sowie ihre reiche Ausstattung führen uns zu dem Schluß, daß man in diesem Großbau ein gegen Ende des vierten Jahrhunderts errichtetes zentrales Gebäude der Burdigala zu sehen hat. Wahrscheinlich handelt es sich um die erste Kathedrale von Bordeaux, die als Teil einer unter der heutigen Kathedrale liegenden Kirchenfamilie errichtet wurde. Zusammen mit den nahegelegenen Basiliken Saint-Seurin und Sainte-Marie belegt dieses eindrucksvolle Bauwerk, wie weit die christlichen Anfänge von Bordeaux zurückreichen.

Übersetzung: Stefan WIRTH

Dans l'angle sud-ouest du castrum antique de Bordeaux, les déplacements de réseaux occasionnés par la mise en place de la plate-forme du tramway ont récemment permis la découverte, sur les places Pey-Berland et Jean-Moulin, de l'abside d'un monument de l'Antiquité tardive que sa proximité $(20 \mathrm{~m})$ avec l'actuelle cathédrale Saint-André invite à interpréter comme un élément du premier groupe épiscopal de la cité (fig. 56). La communauté urbaine de Bordeaux a autorisé des sondages ponctuels au-delà des cotes d'altitude atteintes par les travaux; quelques sondages exploratoires complémentaires avant la réfection de la place ont en outre révélé l'extension et le degré de préservation du monument.

Les relevés stratigraphiques démontrent que l'édifice a été implanté sur des terrains d'origine alluviale, délimités au nord par la vallée de la Devèze. L'abside, semi-circulaire à l'intérieur, présente trois pans coupés à l'extérieur et mesure 13,60 $\mathrm{m}$ de large pour une surface de $119 \mathrm{~m}^{2}$. La largeur de ses murs varie entre $0,85 \mathrm{~m}$ et $1,06 \mathrm{~m}$ entre les pans coupés. Les murs de l'épaulement de l'abside ont une 


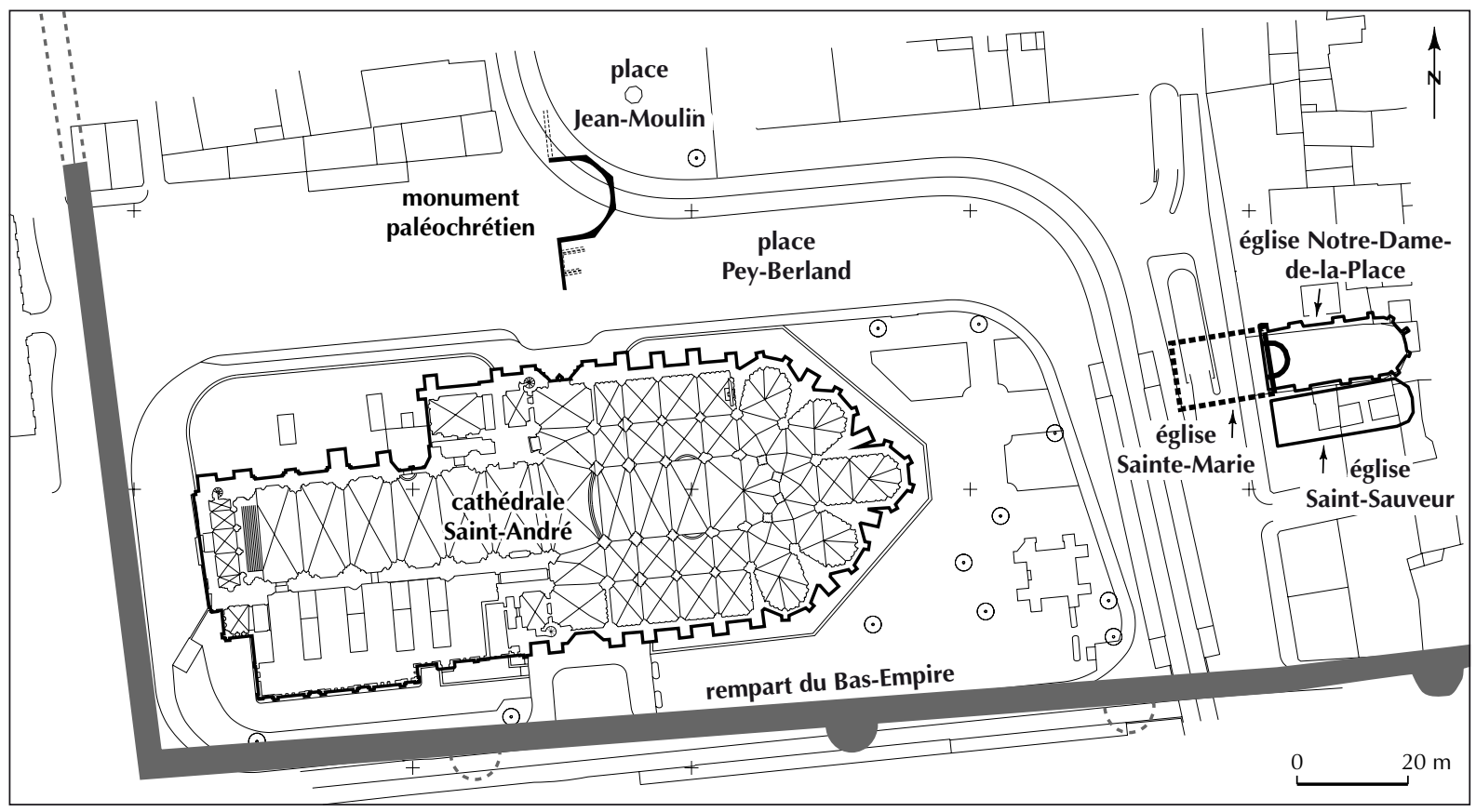

Fig. 56 - Bordeaux (Gironde). Plan de localisation du premier groupe épiscopal identifié dans l'angle sud-ouest du castrum antique (DAO W. Migeon, INRAP).

épaisseur de 0,72 m. L'élévation est constituée par un petit appareil jointoyé au fer; elle était recouverte d'un mortier de chaux de $6 \mathrm{~cm}$ d'épaisseur. Quelques crochets en fer étaient encore fichés dans la maçonnerie. Ils ont dû servir à la fixation d'un placage de marbre blanc, épais de $8 \mathrm{~mm}$, long de 0,30 $\mathrm{m}$ et haut de 0,16 m, dont un élément a été retrouvé en place : il est calé à la base par un morceau de tegula posé dans la préparation du sol, contre le mur, et noyé dans un mortier de chaux.

Le sol est constitué d'un mortier de tuileau épais d'une dizaine de centimètres, qui se situe à 5,80 m NGF. Son radier, composé de petits blocs calcaires et de grave noyés dans un mortier de chaux, prend appui sur le ressaut de fondation du mur de l'abside; il repose sur un remblai de sable blanc contenant en grande quantité des débris de plaquettes de marbre de couleur, de provenances diverses, qui correspondent à des morceaux d'éléments de décors architecturaux en remploi. Un des sondages a cependant mis en évidence un lambeau d'une mosaïque polychrome $(0,70 \mathrm{~m}$ de long sur 0,15 m de large), très abîmée par un violent incendie, qui devait recouvrir l'ensemble du sol intérieur.

L'extérieur, bien que très endommagé au $\mathrm{XI}^{\mathrm{e}} \mathrm{s}$. par la construction d'un bâtiment non identifié, puis au $\mathrm{XV}^{\mathrm{e}} \mathrm{s}$. par la maison du doyen du chapitre de la cathédrale, présentait huit assises d'élévation de petit appareil calcaire
(0,10 m x 0,15 m), ponctuellement réglées par des morceaux de tegulae. Les traces d'un enduit de chaux de $1 \mathrm{~cm}$ d'épaisseur étaient conservées sur plusieurs dizaines de centimètres carrés.

Des sondages complémentaires ont permis de reconnaître partiellement les épaulements de l'abside et d'établir que l'édifice était large d'au moins $28 \mathrm{~m}$, ce qui autorise la restitution d'un transept ou d'un plan basilical à plusieurs nefs. L'extension du bâtiment vers l'ouest est en revanche inconnue, mais la vraisemblance commande de l'imaginer forte d'une bonne quarantaine de mètres. Une pièce de 3,75 m de large et de longueur inconnue était en outre accolée contre l'épaulement sud; ses murs larges de 0,50 m sont parementés de moellons calcaires $(0,10 \mathrm{~m}$ x 0,15 m) disposés en assises régulières et lissés avec un enduit peint rouge.

À la demande du Service régional de l'archéologie, un sondage a percé le sol de tuileau pour rechercher des indices chronologiques. Plusieurs monnaies ont été découvertes, dont une monnaie de Tetricus (antoninien, minimus, vers 270), une monnaie de Maximien Hercule ( $1^{\mathrm{er}}$ règne, antoninien aurelianus [après la réforme d'Aurélien], 5 5 émission, Lyon, 287-289) et une monnaie de Constantin I ${ }^{\text {er }}$ Auguste (308-309, antoninien AE 2 [entre 21 et $22 \mathrm{~mm}$ de diamètre], nummus, buste lauré, drapé à droite, IMP 
CONST(ANTINVS AVG.), buste radié du soleil à droite SOLI INVICTO COM(ITI), pas de marque à l'exergue, atelier de Trèves). Mais la plus récente est une frappe attribuée à un atelier gaulois. Elle porte la légende Decentius Caesar et a été frappée entre 351 et 353, ce qui permet de placer au plus tôt la construction du bâtiment dans la seconde moitié du IV ${ }^{\mathrm{e}}$ s., d'autant qu'à l'extérieur plusieurs niveaux archéologiques venant s'appuyer sur l'abside ont livré des tessons de dérivées de sigillées paléochrétiennes du $\mathrm{V}^{\mathrm{e}} \mathrm{s}$.

Faut-il voir dans ce bâtiment les restes de la première cathédrale de Burdigala où se tint, sans doute en 384, le concile présidé par l'évêque bordelais Delphin, qui condamna le schisme de Priscillien? On restera prudent sur ce point, mais il est sûr que la proximité de l'édifice avec l'actuelle cathédrale Saint-André (qui peut elle-même avoir succédé à un édifice de culte antique) et l'abside d'une autre basilique du VI ${ }^{\mathrm{e}}$ s. que D. Barraud a identifiée en 1983 au-dessous de Notre-Dame-de-la-Place invitent à imaginer à Bordeaux l'existence pendant l'Antiquité tardive d'un groupe épiscopal à la fois complexe et très étendu.

En accord avec la communauté urbaine de Bordeaux, les vestiges ont été conservés au-dessous de la plate-forme du tramway. 